\title{
Applications of Artificial Neural Network in Textiles
}

\author{
Neha Chauhan*, Nirmal Yadav and Nisha Arya \\ Department of Textile and Apparel Designing, I.C. College of Home Science, \\ CCSHAU, Hisar, Haryana, India \\ *Corresponding author
}

\begin{tabular}{|c|}
\hline Keywords \\
\hline $\begin{array}{l}\text { Artificial neural } \\
\text { network, Element }\end{array}$ \\
\hline Article Info \\
\hline $\begin{array}{l}\text { Accepted: } \\
26 \text { March } 2018 \\
\text { Available Online: } \\
10 \text { April } 2018\end{array}$ \\
\hline
\end{tabular}

\section{Introduction}

The artificial neural network (ANN) is an information processing paradigm that is inspired by the way biological nervous system works, such as brain processes an information. ANN uses numeric and associative processing to mimic models of biological system. It resembles the human brain in two respects: i.e. knowledge is acquired by the network from its environment through a learning process and interneuron connection strengths, known as

\section{A B S T R A C T}

An Artificial Neural Network (ANN) is an information processing paradigm that is inspired by the way biological nervous systems, such as the brain, process information. The key element of this paradigm is the novel structure of the information processing system. It is composed of a large number of highly interconnected processing elements (neurones) working in unison to solve specific problems. The artificial neural network is increasingly used as a powerful tool for many real-world problems. In Textiles and Clothing industries, it involves the interaction of a large number of variables. Because of the high degree of variability in raw materials, multistage processing and a lack of precise control on process parameters, the relation between such variables and the product properties is relied on the human knowledge but it is not possible for human being to remember all the details of the process-related data over the years. ANN has proved its usefulness for resolving many problems in textiles such as prediction of yarn properties, analysis of fabric defects, process optimization etc. The power of neural networks lies in their ability to represent complex relationships and learn them directly from the data being modelled. The ability to predict these properties accurately has become a challenge due to highly non-linear and interactive behaviour of textile materials. The prediction of properties or performance of a process in advance is required to minimize the setup cost and time. The function of ANN is not constant but can be changed dynamically. 


\section{Definition}

A set of processing units when assembled in a closely interconnected network, offers a rich structure exhibiting some features of the biological neural network. Such a structure is called as artificial neural network (ANN). The ANN is a massively parallel distributed processor made of single processing units, which has a natural propensity for storing experiential knowledge and make it available for use. The procedure used to perform the learning process is called as learning algorithm, the function of which is to modify the synaptic weights of the network in an orderly fashion to attain a desired design objective. In practice, the ANN cannot provide the solution by working individually, rather it needs to be integrated into a consistent system engineering approach. In 1943, Warren McCulloch and Walter Pits proposed a model of computing element, called as McCulloch Pits neuron. In 1949, Donald Hebb proposed a learning scheme for pre-synaptic and postsynaptic values of variables. In 1958, Rosenblatt proposed the perceptron model, which can adjust weights by perceptron learning law. In 1960, Widraw proposed Adaline model for computing element.

In 1985, Akley, Hinton and Sejnowski proposed feedback neural network with hidden units. For many years, neural networks have been used in various areas of engineering and economics in order to describe the relationship between the parameters that cannot be determined analytically. They were used to simulate learning from examples, detecting patterns, associative memorizing and recalling information.

\section{Classes of neural network}

There are three classes of neural networks: viz. Single layer feedforward networks, Multilayer feedforward networks and
Recurrent networks as shown in Figure 1, 2 and 3 respectively.

\section{Single layer feed forward network}

The neurons are organized in the form of layers. In a simplest form of a layered network, an input layer of source nodes projects onto an outer layer of neurons. The network is strictly feed forward and hence called as single layer feed forward network.

\section{Multilayer feed forward network}

In multilayer network, there is presence of one or more hidden layers whose nodes are called as hidden neurons. Their function is to intervene between external input and network output in some useful manner. By adding more hidden layers, the network is enabled to extract higher order statistics.

The source nodes in input layer supplies respective element of activation pattern, which constitutes input signals applied to neurons in first hidden layer. The output signals of the first hidden layer are used as input to the second hidden layer and so on. The set of output signals of the neurons in the final output layer of network constitutes the overall response of the network to the activation pattern supplied by the source nodes in the first input layer.

\section{Recurrent network}

The recurrent neural network is distinct in the sense that, it has at least one feedback loop. A recurrent network may consist of a single layer of neurons, with each neuron feeding its output signals back to inputs of all other neurons. The recurrent network may or may not have hidden neurons. The presence of feedback loops has a profound impact on learning capability of the network and its performance. 


\section{Learning of a network}

A network can be learnt with or without training. The objective of learning is to mold the mapping surface according to desired response. Once the network is structured for a particular application, that network is ready to be trained. To start with, the initial weights are chosen randomly. There are two approaches to learning: supervised and unsupervised.

\section{Supervised learning}

In this system, both input and output data are provided to network. Then it processes the inputs and compares the resulting outputs against the desired outputs. Errors are propagated backwards through the system, causing system to adjust the weights. The same set of data is processed many times, as the weights are refined. When the system has been fully trained, no further learning is required and the weights can be frozen. A vast bulk of networks utilizes supervised learning.

\section{Unsupervised learning}

This type of network is provided only with inputs. The system itself decides which features to be used to group the input data. It is also called as Adaptive learning. This learning technique is not commercially practiced.

\section{Applications of ANN in textiles}

\section{Fibres}

An Artificial neural network is used by $\mathrm{S}$. Mukhopadhay and Q. Siddiquee for the prediction of copolymer components. The free radical copolymerization of acrylamide with quaternary ammonium cationic co-monomer diethylaminoethyl acrylate (DMAEA) has been investigated in inverse emulsion. The copolymer composition is determined from residual monomer concentration. An ANN is used to predict the copolymer composition. The results shown that, ANN can predict copolymer composition very correctly, as a function of reaction conditions and conversions.

Figure 4 shows the intelligent fibre classification system. Here, ANN is used to classify two kinds of animal fibres; Merino and Mohair. The model extracting six scale parameters with image processing and other using an unsupervised neural network to extract features automatically, which are determined in accordance with the complexity of scale structure and the accuracy of the model. It can achieve higher accuracy by increasing number of samples for learning, since accuracy of ANN largely depends on the parameters selected. Cotton trades in India assess the commercial value of cotton on the basis of "grade" assigned by hand and eye methods by professional cotton classers. Now, ANN can be used to develop models to predict the cotton grading. By this way, cotton grading can be made more reliable. Research is going on in this direction to replace the existing subjective grading with the objective one. To be precise, the properties of cotton that are measured by using ANN are colour of cotton, trash present and ginning preparation. The blend out of two different kinds of fibres, the exact proportion in which the mixing has to be done (to obtain the required properties of the yarn) can be predicted using ANN.

\section{Spinning}

Y.C. Zeng et al., have analysed modeling methods for predicting the tensile properties of air jet spun yarns. Numerical simulation provides a useful insight into a relationship between yarn tenacity and parameters. The neural network model predicts yarn tenacity using input parameters. The predicted and experimental values agreed well, indicating 
that the neural network is the excellent method for predictors. They also analyzed the effect of input parameters on yarn tenacity with neural network model. M. C. Ramesh et al., predicted the tensile properties of yarn from fibre properties. The feasibility of yarn engineering is demonstrated in Figure 5 by developing a yarn to fibre "reverse" model using ANN. This approach is entirely different from the prevailing forward models, which predict the properties of final yarn by using the fibre properties as inputs. The cost minimization of cotton fibre was ensured by using classical linear programming approach in combination with ANN. The engineered yarns demonstrated good agreement with the targeted yarn properties. Spinning consistency index (SCI) was chosen for this purpose, as the regression equation of this index comprises most of cotton properties.

Luo. Cheng and David Adams used an ANN for prediction of relationship between fibre properties and yarn strength. A three-layer feed forward network along with a correlation regression is used. They used USDA cotton fibre and processing test results for this investigation. The fibre properties like upper half mean length, length uniformity, short fibre content, strength; fineness and maturity ratio are measured using HVI. All cotton samples were spun into 22s ring spun yarn. Yarns were tested for skein test, elongation, appearance and imperfection. The CSP was used as a prediction target. When the neural networks were trained by a training set CSP values could be predicted from the neural networks in the prediction mode. Then the predicted CSP were correlated with the actual CSP and a good agreement is found to be existing between fibre properties and yarn strength.

Chung Feng Jeffrey et al., studied the relationship between process variables and molecular structure. The thermo-mechanical properties of PET yarns were determined using ANN. Although the internal structure of ANN is incomprehensive with respect to mechanisms of modelled relations, it was accurate way to make data and relations between data sets easily accessible. Das A. used a multilayer feed forward network with back propagation algorithm to determine the optimized machine settings of a draw texturing machine, with regard to desired yarn properties. The properties of yarn like tenacity and elongation are predicted by using regression analysis and the process parameters used are draw ratio, $\mathrm{D} / \mathrm{Y}$ ratio, speed and primary heater temperature.

Traditionally, the quality grades of false twist yarn packages are classified by human inspection, but the result may be affected by personal and subjective factors. Shih-Hsuan Chu et al., used the image processing technology to extract the defects in yarn packages. They used neural networks to classify the quality grades of the yarn packages. From the experimental results, they obtained the classifying rate of about $90 \%$.

\section{Knitting}

S. Ertugrul and N Ucar studied the prediction of bursting strength of knitted fabrics. Bursting strength of cotton plain knitted fabrics is predicted before manufacturing by an intelligent technique of neural network and neuro fuzzy approaches, by achieving a significant savings in cost and time required for trial and error. Among many parameters that affect fabric bursting strength; the fabric weight, yarn breaking strength and elongation are the input elements for this prediction. Prediction of total hand value of knits is reported to be successful by Park. A fuzzy neural network provides an effective tool for prediction of total hand value of outerwear knit fabrics. A fuzzy neural network is developed to predict and display the drope 
images of garments made of different fabrics and styles. The new approach is used to develop a prototype drope prediction system, to predict drope of women's dress styles made from different fabrics.

\section{Weaving}

Furferi et al., (2012) presented a work to provide a predictive model of a coating process for forecasting the final characteristics of a coated fabric, based on the process parameters. The ANN is trained and validated using a wide experimental database. The work is proved to be useful for technicians in selecting the optimal processing parameters for obtaining the desired coated fabric properties. The developed system requires technicians to set the values of process parameters and to stimulate the ANN based model to forecast the quality of coated fabric. An image processing system is used by Atiqul Islam et al., as a tool for dynamic inspection of fabrics; the inspection sample is a piece of plain white fabric. The four defects are holes, oil stains, warp lacking and weft lacking. The image treatment employs a high resolution linear scan digital camera. Fabric images are acquired first, and then the images are transferred to a computer for analysis. Finally, the data is adopted as input for neural network which is obtained from readings after treating the images. In this system, there are three feed forward networks; an input layer, one hidden layer and an outer layer. As it has the ability to cope with the non-linear regression property, this method can reinforce the effects of image identification. Automatic recognition of woven fabric patterns presented by Boong et $a l$., The neural network and image processing technology are introduced for classifying woven fabric patterns. An auto correlation function is used to determine one weave repeat of the fabric. The reflected fabric image is captured by CCD camera and digitized by computer system. The learning vector quantization's algorithm as a learning rule of the artificial neural network enables recognition of woven fabric types more effectively. The results demonstrated that, the three fundamental weaves can be classified accurately and structural parameters such as yarn spacing, its variance and the ratio of warp to weft spacing can also be obtained. The system consists of a PC and CCD (charged coupled device) camera equipped with an attachable zoom lens. The fabric image is captured by a lightening device which is mounted at the end of the camera and zooms in on the image, which is also displayed on the coloured monitor. The image is then digitised by the AC to DC convertor and processed using techniques such as thresholding and auto-correlation to analyse the fabric pattern. The original image is converted into a grey level image to improve computer processing time and speed and the histograms are obtained.

Jasper W. J. used the back-propagation algorithm to study the missing ends and picks, oily fabrics and broken fabric, all often found as a defect in fabric. The correct selection of parameters for input layer plays a great role in recognition rate. Once a defect occurs in a fabric, its periodicity is changed so that corresponding intensities at specific position of spectrum obviously changed. These intensions can act as characteristics parameters and can be substituted in ANN for learning. In a study by Tsai and $\mathrm{Hu}$, the efficiency and accuracy of a method of detecting fabric defects that have been classified into different categories by neural network. Four fabric defects most likely found during weaving were learned by network. The method used for processing image is cooccurrence-based method, by which six parameters are obtained. The results show that, the fabric defects inspected by means of image reorganization in accordance with ANN agrees with initial expectations. 


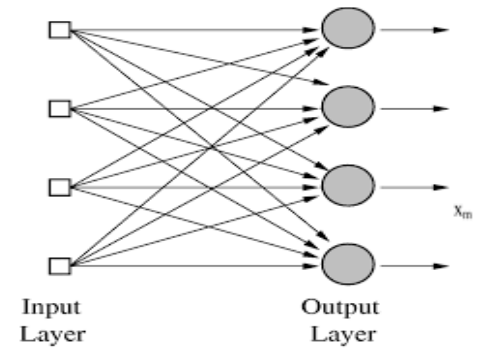

Fig.1. Single layer NN

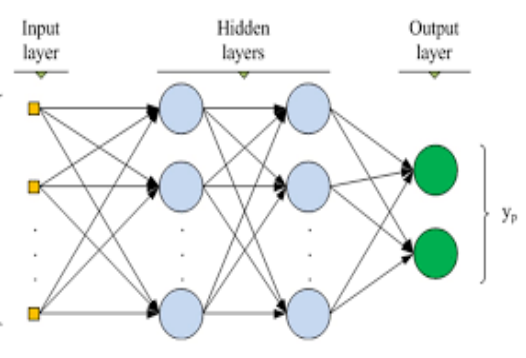

Fig.2. Multi-layer NN

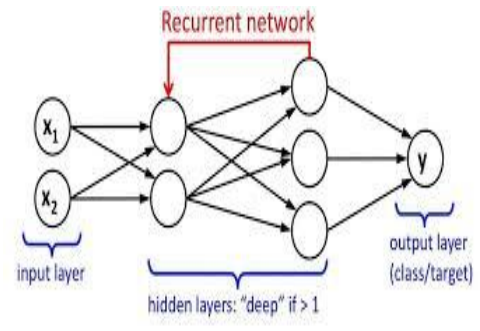

Fig.3. Recurrent NN

Fig.4 Intelligent fibre classification system

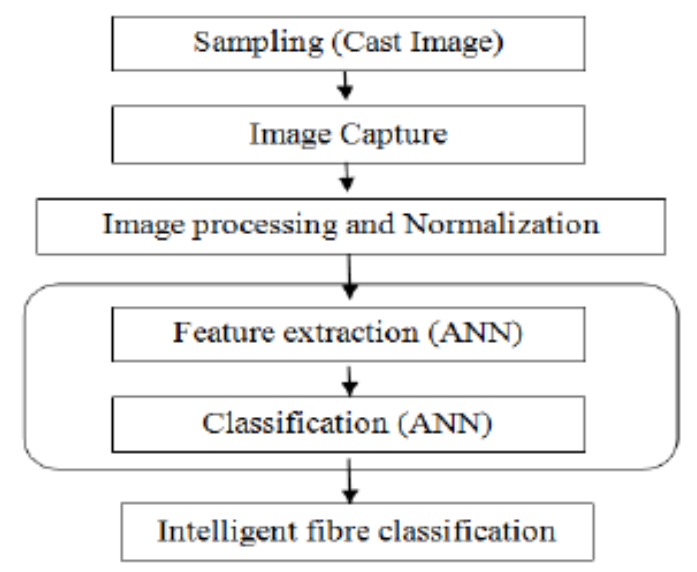

Fig.5 ANN model for yarn engineering

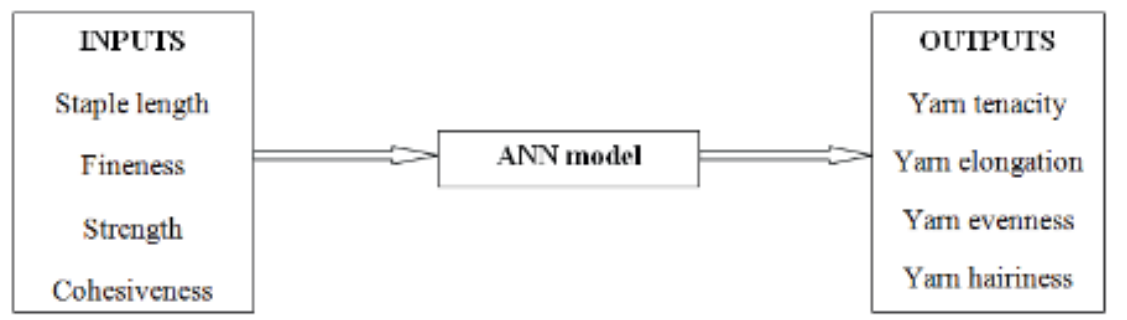

Fig.6 Uster Fabriscan

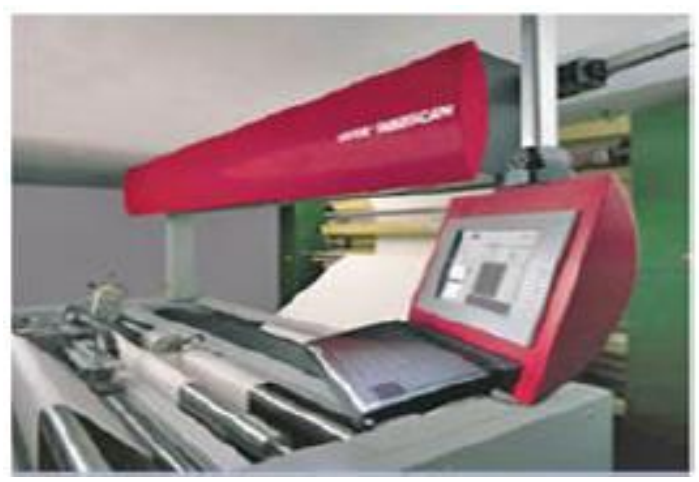


Fig.7 Neural network for fabric engineering

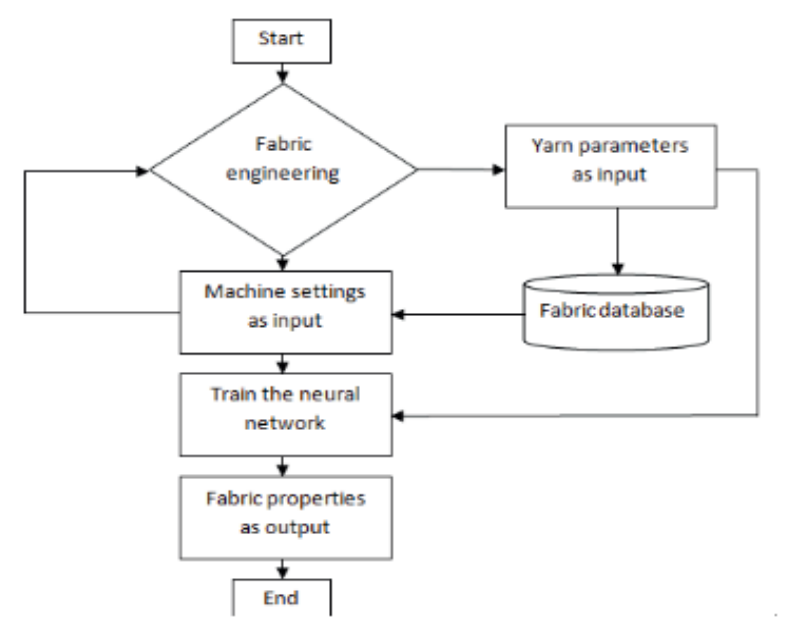

The Uster fabric scan system uses ANN in evaluating wrinkled fabrics with image analysis. Wrinkles in cloth usually develop with deformation during storage. It is not easy even for trained observers judge the wrinkles. Here an objective method is being proposed. The visual information stimulates their senses and makes them to judge the grade of wrinkle. Since visual evaluation is complex and bias, we cannot use linear evaluation system (Fig. 7).

For auto inspection of wrinkles in fabric, neural networks are widely used. Since networks are non-linear, the training rule is regularized as non-linear optimization problem. Input parameters such as angular second monument, contrast, correlation, entropy and fractal dimension are obtained using image analysis and are fed to the neural network algorithm. The mean sensory value presenting the grade of wrinkled fabric as output is obtained with desired parameters. The parameters to be perceived such as density, size, form and contrast can be inspected using multi-input and multi-output concept of neural network. A specific algorithm named "Kalman's Filter Algorithm" is used for this purpose and results are referred to be very much feasible for evaluating wrinkled fabrics. The relationship between structure and properties of the fabric is complex and inherently non-linear; to create a predictive model one must resolve these complexities. The optimization of input parameters required to produce a fabric as per end use is called as fabric engineering. Fabrics can be engineered either by weaving, knitting or bonding neural networks are successfully implemented in all three to optimize the input parameters. The yarn properties and machine settings are used as inputs to predict the fabric properties as outputs. The construction of neural network for fabric engineering is shown in Figure 7.

In prediction of human psychological perceptions of clothing sensory comfort ANN is widely used. The objective is to know the predictability of the clothing sensory comfort from psychological perceptions by using feed forward back propagation network in ANN system. In order to achieve the objective, a series of wear trials are conducted in which 10 sensory perceptions (clammy, clingy, damp, sticky, heavy, prickly, scratchy, fit, breathable and thermal) and overall clothing comfort is rated. Good agreement between predicted and actual clothing comfort perceptions proved that, the neural network is effective technique for modelling the psychological perceptions of clothing sensory 
comfort. It is also found that, the predicted comfort score from the model with hidden neurons and the linear output neuron has a better fit with the actual comfort score than other models with different combinations of hidden and output neurons. Compared with statistical modelling techniques, the neural network is a fast and flexible predictive tool with a self-learning ability for clothing comfort perceptions.

\section{Color}

The color measurement, comparison, evaluation and prediction are major actions in the dyeing and finishing field of the textile process. Although color measurement is possible in the laboratory with the help of specialized equipment like the spectrophotometers, few capabilities exist for the prediction of the color changes or the final color appearance because the problem is multivariable. A model for the prediction of color change after the spinning process has been developed. The prediction of the color and the color solidity of a jigger dyed cellulose based fabric has been achieved by using cascade ANNs. In the field of printing, the color recipe specification has been made possible using radial basis function neural networks. The pigment combinations for the textile printing can be determined, the color of the printed fabric images can be identified and the color separation can take place by using different ANN types.

The prediction of CIELAB values is possible for color changes after chemical processes, for nylon 6, 6 and for stripped cotton fabrics. The optimization of the processing conditions and the prediction of the dyeing quality of nylon and lycra fabrics and the classification of dyeing defects have been carried out with the help of ANNs and fuzzy neural networks respectively.

\section{Nonwovens}

The nonwoven is a specific category of fabrics, made directly of fibres and not of yarns. Then on woven fabrics find many technical applications and their role is essential. Then on woven fabrics undergo a process of inspection in order to ensure quality of the delivered material. A visual inspection system has been based on wavelet texture analysis and robust Bayesian ANNs, or similarly wavelet transforms and ANNs, while a neuro-fractal approach has been used for the recognition and classification of non-woven web images. Many quality issues are addressed via ANN methods, like the structure-properties relations of the nonwoven fabrics, the construction of a quality prediction system, the modelling of the compression properties of needle-punched nonwoven fabrics, the simulation of the drawing of spun bonding now oven process and also the objective evaluation of the pilling on nonwoven fabrics.

\section{Making up and clothing}

Clothing articles are the end product of the main stream of the textile production flow. Although precision of the prediction of properties is not that critical as in technical applications, estimation of the final properties is essential for the clothing design, the selection of raw materials and their required properties. One of the most important factors affecting the garment quality is related to the seam, the result of the sewing process. Indeed, prediction of the seam strength is very important, especially for parachutes. The thread consumption is predicted via an ANN model, while the seam puckering is evaluated and the sewing thread is optimized through ANN models, respectively. The prediction of the sewing performance is also possible using ANNs. The human psychological perceptions of the clothing sensory comfort and the analysis of the tactile perception of the textile materials can be carried out using ANN approaches. Prediction of the performance of the fabrics in garment manufacturing and fit garment design has been realized based on ANN systems. Cases of special interest, like the selection of the optimal interlinings, or of broad interest, like the simulation of a textile supply chain, have been successfully modelled by ANNs. 


\section{Applications to chemical processing}

Huang and $\mathrm{Yu}$ used image processing and fuzzy neural network approaches to classify seven kinds of dyeing defects including filling band in shade, dye and carrier spots, mist, oil stain, tailing, listing, and uneven dyeing on selvage. The fuzzy neural classification system was constructed by a fuzzy expert system with the neural network as a fuzzy inference engine so it was more intelligent in handling pattern recognition and classification problems. The neural network was trained to become the inference engine using sample data. Region growing was adopted to directly detect different defect regions in an image. Seventy samples, ten samples for each defect, were obtained for training and testing. The results demonstrated that the fuzzy neural network approach could precisely classify the defective samples by the features selected.

Potential future application of ANN in textiles and clothing industries

A large number of applications of ANN in textiles and clothing industries are used feed forward and Kohonen networks. The other types of artificial neural networks such as recurrent neural network, associative neural network and dynamic neural networks are rarely used. Meanwhile, quite a few areas remains insufficiently explored such as knitting, nonwoven fabrics and finishing control. Exploring such areas using new ANN models is anew trend in future research. In the future research, the following issues shall be taken into consideration to the application of ANN in textiles and clothing industries.

Improves the data collection method for training ANNs such as online data captured from the process

Improves the feature-extraction procedures before the data can be fed to an ANN

Improves extrapolation ability of the system to strengthen the prediction capability
Improves the user-friendly interface between user and machine

These issues are important for further development of using ANN in textiles and clothing industries. Further research works shall deal with such issues in order to set up intelligent systems in textiles and clothing fields instead of human judgment.

ANN is field of computer-based technology that can simulate characteristics of human interference for industrial benefits. ANN aims at the requirements of the industry like least downtime, highly competitiveness and reliable estimates of properties and process parameters. The function of ANN is not constant but can be changed dynamically. Neural network technique is used to model non-linear problems and predict the output values for given input parameters. Most of the textile processes and the related quality assessments are non-linear in nature and hence, neural networks find application in textile technology. ANN may be defined as structures comprised of densely interconnected adaptive simple processing elements that are capable of performing massively parallel computations for data processing and knowledge representation. There are many different types of neural networks varying fundamentally. The most commonly used type of ANN in textile industry is the multi-layered perceptron (MLP) trained neural network. MLP is a feed-forward neural network. In most textile applications a feedforward network with a single layer of hidden units is used with a sigmoid activation function for the units. The ANN model was found to be more accurate than multiple regression and the prediction errors of ANN were low despite the availability of only a small training data set. The number of input and output neurons depends on the type of textile problems. Many of the techniques reported require many feature extraction procedures before the data can feed to a neural network and data is afforded by different measurements including feature extracted from images, experiments based on standards based on their own tests or other 
gathered measurements. Sum of the square error (SSE), moment correlation coefficient (r), percentage error $(\% \mathrm{E})$, coefficient of variation $(\% \mathrm{CV})$, gamma factor $(\gamma)$, performance factor $(\mathrm{PF} / 4)$, and etc. in order to analyse the results. Since neural networks are known to be good at solving classification problems, it is not surprising that much research has been done in the area of textile classification, particularly fault identification and classification. The current 2D-based investigation needs to be extended to 3D space for actual manual inspection.

\section{References}

Azim G. A. 2015. Identification of Textile Defects Based on GLCM and Neural Networks. Journal of Computer and Communications. 3. 1-8.

Farooq A., and Cherif C. 2008. Use of Artificial Neural Networks for Determining the Leveling Action Point at the Autoleveling Draw Frame. Textile Research Journal. 78(6). 502-509.

Furferi R., and Carfagni M. 2010. Prediction of the Color and the Color Solodity of a Jigger-dyed Cellulose-based Fabric: A Cascade Neural Network Approach. Textile Research Journal. 80(16). 16821696.

Furferi R., Governi L., and Volpe Y. 2012. Modelling and simulation of an innovative fabric coating process using artificial neural networks. Textile Research Journal. 82(12). 1282-1294.

Hui C., and Ng S. 2009. Predicting Seam Performance of Commercial Woven
Fabrics Using Multiple Logarithm Regression and Artificial Neural Networks. Textile Research Journal. 79(18). 1649-1657.

Hui C., Fun N., and Ip C. 2011. Review of Application of Artificial Neural Networks in Textiles and Clothing Industries over Last Decades. Book: Artificial Neural Networks - Industrial and Control Engineering Applications.

Jawahar N., Kannan C.B.N., Manobhai M.K. 2015. Artificial neural networks for colour prediction in leather dyeing on the basis of a tristimulus system. Color Technology. 131(1). 48-57.

Kang T. J. and Kim, S. C. 2011. Objective Evaluation of the Trash and Color of Raw Cotton by Image Processing and Neural Network. Textile Research Journal. 81(9). 776-782.

Kumar C. and Dhinakaran M. 2015. Scope for Artificial Neural Network in Textiles. IOSR Journal of Polymer and Textile Engineering. 2(1). 34-39.

Liu J., Zuo B., Zeng X., Vroman P., Rabenosolo B. and Bai L. 2010. Visual Quality Recognition of Nonwovens using Wavelet Texture Analysis and Robust Bayesian Neural Network. Textile Research Journal. 80(13). 1278-1289.

Liu J., Zuo B., Zeng X., Vroman P., Rabenosolo B. and Zhang G. 2010. A comparison of robust Bayesian and LVQ neural network for visual conformity recognition of nonwovens. Textile Research Journal. 81(8). 763-777.

\section{How to cite this article:}

Neha Chauhan, Nirmal Yadav and Nisha Arya. 2018. Applications of Artificial Neural Network in Textiles. Int.J.Curr.Microbiol.App.Sci. 7(04): 3134-3143. doi: https://doi.org/10.20546/ijcmas.2018.704.356 